\title{
Twelve Definitions of a Stable Model
}

\author{
Vladimir Lifschitz \\ Department of Computer Sciences, University of Texas at Austin, USA \\ vl@cs.utexas.edu
}

\begin{abstract}
This is a review of some of the definitions of the concept of a stable model that have been proposed in the literature. These definitions are equivalent to each other, at least when applied to traditional Prologstyle programs, but there are reasons why each of them is valuable and interesting. A new characterization of stable models can suggest an alternative picture of the intuitive meaning of logic programs; or it can lead to new algorithms for generating stable models; or it can work better than others when we turn to generalizations of the traditional syntax that are important from the perspective of answer set programming; or it can be more convenient for use in proofs; or it can be interesting simply because it demonstrates a relationship between seemingly unrelated ideas.
\end{abstract}

\section{Introduction}

This is a review of some of the definitions, or characterizations, of the concept of a stable model that have been proposed in the literature. These definitions are equivalent to each other when applied to "traditional rules" - with an atom in the head and a list of atoms, some possibly preceded with the negation as failure symbol, in the body:

$$
A_{0} \leftarrow A_{1}, \ldots, A_{m}, \text { not } A_{m+1}, \text { not } A_{n} .
$$

But there are reasons why each of them is valuable and interesting. A new characterization of stable models can suggest an alternative picture of the intuitive meaning of logic programs; or it can lead to new algorithms for generating stable models; or it can work better when we turn to generalizations of the traditional syntax that are important from the perspective of answer set programming (ASP); or it can be more convenient for use in proofs, such as proofs of correctness of ASP programs; or, quite simply, it can intellectually excite us by demonstrating a relationship between seemingly unrelated ideas.

We concentrate here primarily on programs consisting of finitely many rules of type (1), although generalizations of this syntactic form are mentioned several times in the second half of the paper. Some work on the stable model semantics (for instance, [Gelfond and Lifschitz, 1990], [Lifschitz et al., 1999], [Niemelä and Simons, 2000], [Balduccini and Gelfond, 2003]) is not discussed here simply because it is about extending, rather than modifying, the definitions proposed earlier; this kind of work does not tell us much new about stable models of traditional programs. 
The paper begins with comments on the relevant work that had preceded the invention of stable models - on the semantics of logic programming (Section 2) and on formal nomonotonic reasoning (Section 3). Early contributions that can be seen as characterizations of the class of stable models in terms of nonmonotonic logic are discussed in Section 4. Then we review the definition of stable models in terms of reducts (Section 5) and turn to its characterizations in terms of unfounded sets and loop formulas (Section 6). After that, we talk about three definitions of a stable model that use translations into classical logic (Sections 7 and 8) and about the relation between stable models and equilibrium logic (Section 9).

In recent years, two interesting modifications of the definition of the reduct were introduced (Section 10). And we learned that a simple change in the definition of circumscription can give a characterization of stable models (Section 11).

\section{Minimal Models, Completion, and Stratified Programs}

\subsection{Minimal Models vs. Completion}

According to van Emden and Kowalski [1976], a logic program without negation represents the least (and so, the only minimal) Herbrand model of the corresponding set of Horn clauses. On the other hand, according to Clark [1978], a logic program represents a certain set of first-order formulas, called the program's completion.

These two ideas are closely related to each other, but not equivalent. Take, for instance, the program

$$
\begin{aligned}
& p(a, b) . \\
& p(X, Y) \leftarrow p(Y, X) .
\end{aligned}
$$

The minimal Herbrand model

$$
\{p(a, b), p(b, a)\}
$$

of this program satisfies the program's completion

$$
\forall X Y(p(X, Y) \leftrightarrow((X=a \wedge Y=b) \vee p(Y, X))) \wedge a \neq b .
$$

But there also other Herbrand interpretations satisfying the program's completionfor instance, one that makes $p$ identically true.

Another example of this kind, important for applications of logic programming, is given by the recursive definition of transitive closure:

$$
\begin{aligned}
& q(X, Y) \leftarrow p(X, Y) . \\
& q(X, Z) \leftarrow q(X, Y), q(Y, Z) .
\end{aligned}
$$

The completion of the union of this program with a definition of $p$ has, in many cases, unintended models, in which $q$ is weaker than the transitive closure of $p$ that we want to define. 
Should we say then that Herbrand minimal models provide a better semantics for logic programming than program completion? Yes and no. The concept of completion has a fundamental advantage: it is applicable to programs with negation. Such a program, viewed as a set of clauses, usually has several minimal Herbrand models, and some of them may not satisfy the program's completion. Such "bad" models reflect neither the intended meaning of the program nor the behavior of Prolog. For instance, the program

$$
\begin{aligned}
& p(a) . \quad p(b) . \quad q(a) . \\
& r(X) \leftarrow p(X), \text { not } q(X) .
\end{aligned}
$$

has two minimal Herbrand models:

$$
\{p(a), p(b), q(a), r(b)\}
$$

("good") and

$$
\{p(a), p(b), q(a), q(b)\}
$$

("bad"). The completion of (4)

$$
\begin{gathered}
\forall X(p(X) \leftrightarrow(X=a \vee X=b)) \wedge \forall X(q(X) \leftrightarrow X=a) \\
\wedge \forall X(r(X) \leftrightarrow(p(X) \wedge \neg q(X))) \wedge a \neq b
\end{gathered}
$$

characterizes the good model.

\subsection{The Challenge}

In the 1980s, the main challenge in the study of the semantics of logic programming was to invent a semantics that

- in application to a program without negation, such as (2), describes the minimal Herbrand model,

- in the presence of negation, as in example (4), selects a "good" minimal model satisfying the program's completion.

Such a semantics was proposed in two papers presented at the 1986 Workshop on Foundations of Deductive Databases and Logic Programming [Apt et al., 1988], [Van Gelder, 1988]. That approach was not without defects, however. First, it is limited to programs in which recursion and negation "don't mix." Such programs are called stratified. Unfortunately, some useful Prolog programs do not satisfy this condition. For instance, we can say that a position in a twoperson game is winning if there exists a move from it to a non-winning position (cf. [van Emden and Clark, 1984]). This rule is not stratified: it recursively defines winning in terms of non-winning. A really good semantics should be applicable to rules like this.

Second, the definition of the semantics of stratified programs is somewhat complicated. It is based on the concept of the iterated least fixpoint of a program, and to prove the soundness of this definition one needs to show that this fixpoint 
doesn't depend on the choice of a stratification. A really good semantics should be a little easier to define.

The stable model semantics, as well as the well-founded semantics [Van Gelder et al., 1988, 1991], can be seen as an attempt to generalize and simplify the iterated fixpoint semantics of stratified programs.

\section{$3 \quad$ Nonmonotonic Reasoning}

Many events in the history of research on stable models can be only understood if we think of it as part of a broader research effort - the investigation of nonmonotonic reasoning. Three theories of nonmonotonic reasoning are particularly relevant.

\subsection{Circumscription}

Circumscription [McCarthy, 1980, 1986] is a syntactic transformation that turns a first-order sentence $F$ into the conjunction of $F$ with another formula, which expresses a minimality condition (the exact form of that condition depends on the "circumscription policy"). This additional conjunctive term involves secondorder quantifiers.

Circumscription generalizes the concept of a minimal model from [van Emden and Kowalski, 1976]. The iterated fixpoint semantics of stratified programs can be characterized in terms of circumscription also [Lifschitz, 1988]. On the other hand, circumscription is similar to program completion in the sense that both are syntactic transformations that make a formula stronger. The relationship between circumscription and program completion was investigated by Reiter [1982].

\subsection{Default Logic}

A default theory in the sense of [Reiter, 1980] is characterized by a set $W$ of "axioms" - first-order sentences, and a set $D$ of "defaults" - expressions of the form

$$
\frac{F: \mathrm{M} G_{1}, \ldots, \mathrm{M} G_{n}}{H},
$$

where $F, G_{1}, \ldots, G_{n}, H$ are first-order formulas. The letter $\mathrm{M}$, according to Reiter, is to be read as "it is consistent to assume." Intuitively, default (7) is similar to the inference rule allowing us to derive the conclusion $H$ from the premise $F$, except that the applicability of this rule is limited by the justifications $G_{1}, \ldots, G_{n}$; deriving $H$ is allowed only if each of the justifications can be "consistently assumed."

This informal description of the meaning of a default is circular: to decide which formulas can be derived using one of the defaults from $D$ we need to know whether the justifications of that default are consistent with the formulas that can be derived from $W$ using the inference rules of classical logic and the defaults from $D$-including the default that we are trying to understand! But 
Reiter was able to turn his intuition about $M$ into a precise semantics. His theory of defaults tells us under what conditions a set $E$ of sentences is an "extension" for the default theory with axioms $W$ and defaults $D$.

In Section 4 we will see that one of the earliest incarnations of the stable model semantics was based on treating rules as defaults in the sense of Reiter.

\subsection{Autoepistemic Logic}

According to [Moore, 1985], autoepistemic logic "is intended to model the beliefs of an agent reflecting upon his own beliefs." Moore's definition of propositional autoepistemic logic builds on the ideas of [McDermott and Doyle, 1980] and [McDermott, 1982].

Formulas of this logic are constructed from atoms using propositional connectives and the modal operator L ("is believed"). Its semantics specifies, for any set $A$ of formulas ("axioms"), which sets of formulas are considered "stable expansions" of $A$. Intuitively, Moore explains, the stable expansions of $A$ are "the possible sets of beliefs that a rational agent might hold, given $A$ as his premises."

In Section 4 we will see that one of the earliest incarnations of the stable model semantics was based on treating rules as autoepistemic axioms in the sense of Moore. The term "stable model" is historically related to "stable expansions" of autoepistemic logic.

\subsection{Relations between Nonmonotonic Formalisms}

The intuitions underlying circumscription, default logic, and autoepistemic logic are different from each other, but related. For instance, circumscribing (that is, minimizing the extent of) a predicate $p$ is somewhat similar to adopting the default

$$
\frac{\text { true }: \mathrm{M} \neg p(X)}{\neg p(X)}
$$

(if it is consistent to assume that $X$ does not have the property $p$, conclude that it doesn't). On the other hand, Moore observes that "a formula is consistent if its negation is not believed"; accordingly, Reiter's M is somewhat similar to the combination $\neg \mathrm{L} \neg$ in autoepistemic logic, and default (7), in propositional case, is somewhat similar to the autoepistemic formula

$$
F \wedge \neg \mathrm{L} \neg G_{1} \wedge \cdots \wedge \neg \mathrm{L} \neg G_{n} \rightarrow H .
$$

However, the task of finding precise and general relationships between these three formalisms turned out to be difficult. Discussing technical work on that topic is beyond the scope of this paper. 


\section{Definitions A and B, in Terms of Translations into Nonmonotonic Logic}

The idea of [Gelfond, 1987] is to think of the expression not $A$ in a logic program as synonymous with the autoepistemic formula $\neg \mathrm{L} A$ ( " $A$ is not believed"). Since autoepistemic logic is propositional, the program needs to be grounded before this transformation is applied. After grounding, each rule (1) is rewritten as a formula:

$$
A_{1} \wedge \cdots \wedge A_{m} \wedge \neg A_{m+1} \wedge \cdots \wedge \neg A_{n} \rightarrow A_{0},
$$

and then $L$ inserted after each negation. For instance, to explain the meaning of program (4), we take the result of its grounding

$$
\begin{aligned}
& p(a) . \quad p(b) . \quad q(a) . \\
& r(a) \leftarrow p(a), \text { not } q(a) . \\
& r(b) \leftarrow p(b), \text { not } q(b) .
\end{aligned}
$$

and turn it into a collection of formulas:

$$
\begin{aligned}
& p(a), \quad p(b), \quad q(a), \\
& p(a) \wedge \neg \mathrm{L} q(a) \rightarrow r(a), \\
& p(b) \wedge \neg \mathrm{L} q(b) \rightarrow r(b) .
\end{aligned}
$$

The autoepistemic theory with these axioms has a unique stable expansion, and the atoms from that stable expansion form the intended model (5) of the program.

This epistemic interpretation of logic programs - what we will call Definition $A$-is more general than the iterated fixpoint semantics, and it is much simpler. One other feature of Definition A that makes it attractive is the simplicity of the underlying intuition: negation as failure expresses the absence of belief.

The "default logic semantics" proposed by Bidoit and Froidevaux [1987] is translational as well; it interprets logic programs as default theories. The head $A_{0}$ of a rule (1) turns into the conclusion of the default, the conjunction $A_{1} \wedge \cdots \wedge A_{m}$ of the positive members of the body becomes the premise, and each negative member not $A_{i}$ turns into the justification $\mathrm{M} \neg A_{i}$ ("it is consistent to assume $\neg A_{i}$ "). For instance, the last rule of program (4) corresponds to the default

$$
\frac{p(X): \mathrm{M} \neg q(X)}{r(X)} .
$$

There is no need for grounding, because defaults are allowed to contain variables. This difference between the two translations is not essential though, because Reiter's semantics of defaults treats a default with variables as the set of its ground instances. Grounding is simply "hidden" in the semantics of default logic.

This Definition $B$ of the stable model semantics stresses an analogy between rules in logic programming and inference rules in logic. Like Definition A, it 
has an epistemic flavor, because of the relationship between the "consistency operator" $M$ in defaults and the autoepistemic "belief operator" L (Section 3.4).

The equivalence between these two approaches to semantics of traditional programs follows from the fact that each of them is equivalent to Definition $\mathrm{C}$ of a stable model reviewed in the next section. This was established in [Gelfond and Lifschitz, 1988] for the autoepistemic semantics and in [Marek and Truszczyński, 1989] for the default logic approach.

\section{Definition C, in Terms of the Reduct}

Definitions A and B are easy to understand - assuming that one is familiar with formal nonmonotonic reasoning. Can we make these definitions direct and avoid explicit references to autoepistemic logic and default logic?

This question has led to the most widely used definition of the stable model semantics, Definition $C$ [Gelfond and Lifschitz, 1988]. The reduct of a program $\Pi$ relative to a set $M$ of atoms is obtained from $\Pi$ by grounding followed by

(i) dropping each rule (1) containing a term not $A_{i}$ with $A_{i} \in M$, and

(ii) dropping the negative parts not $A_{m+1}, \ldots$, not $A_{n}$ from the bodies of the remaining rules.

We say that $M$ is a stable model of $\Pi$ if the minimal model of (the set of clauses corresponding to the rules of) the reduct of $\Pi$ with respect to $X$ equals $X$. For instance, the reduct of program (4) relative to (5) is

$$
\begin{aligned}
& p(a) . \quad p(b) . \quad q(a) . \\
& r(b) \leftarrow p(b) .
\end{aligned}
$$

The minimal model of this program is the set (5) that we started with; consequently, that set is a stable model of (4).

Definition C was independently invented by Fine [1989].

\section{Definitions D and E, in Terms of Unfounded Sets and Loop Formulas}

Saccá and Zaniolo [1990] showed that stable models can be characterized in terms of the concept of an unfounded set, which was introduced in [Van Gelder et al., 1988] as part of the definition of the well-founded semantics. Namely, a set $M$ of atoms is a stable model of a (grounded) program $\Pi$ iff

(i) $M$ satisfies $\Pi,{ }^{1}$ and

(ii) no nonempty subset of $M$ is unfounded for $\Pi$ with respect to $M{ }^{2}$

${ }^{1}$ That is, $M$ satisfies the propositional formulas (8) corresponding to the rules of $\Pi$.

2 To be precise, unfoundedness is defined with respect to a partial interpretation, not a set of atoms. But we are only interested here in the special case when the partial interpretation is complete, and assume that complete interpretations are represented by sets of atoms in the usual way. 
According to Lee [2005], this Definition D can be refined using the concept of a loop, introduced many years later by Lin and Zhao [2004]. If we require, in condition (i), that $M$ satisfy the completion of the program, rather than the program itself, then it will be possible to relax condition (ii) and require only that no loop contained in $M$ be unfounded; there will be no need then to refer to arbitrary nonempty subsets in that condition.

Lin and Zhao used loops in a different way. They associated with every loop $X$ of $\Pi$ a certain propositional formula, called the loop formula for $X$. According to their Definition $E, M$ is a stable model of $\Pi$ iff $M$ satisfies the completion of $\Pi$ conjoined with the loop formulas for all loops of $\Pi$.

The invention of loop formulas has led to the creation of systems for generating stable models that use SAT solvers for search ("SAT-based answer set programming"). Several systems of this kind performed well in a recent ASP system competition [Gebser et al., 2007].

\section{Definition F, in Terms of Circumscription}

We saw in Section 4 that a logic program can be viewed as shorthand for an autoepistemic theory or a default theory. The characterization of stable models described in [Lin, 1991, Section 3.4.1] relates logic programs to the third nonmonotonic formalism reviewed above, circumscription. Like Definitions A and B, it is based on a translation, but the output of that translation is not simply a circumscription formula; it involves also some additional conjunctive terms.

The first step of that translation consists in replacing the occurrences of each predicate symbol $p$ in the negative parts $\neg A_{m+1} \wedge \cdots \wedge \neg A_{n}$ of the formulas (8) corresponding to the rules of the program with a new symbol $p^{\prime}$ and forming the conjunction of the universal closures of the resulting formulas. The sentence obtained in this way is denoted by $C(\Pi)$. For instance, if $\Pi$ is $(4)$ then $C(\Pi)$ is

$$
p(a) \wedge p(b) \wedge q(a) \wedge \forall X\left(p(X) \wedge \neg q^{\prime}(X) \rightarrow r(X)\right) .
$$

The translation of $\Pi$ is a conjunction of two sentences: the circumscription of the old (non-primed) predicates in $C(\Pi)$ and the formulas asserting, for each of the new predicates, that it is equivalent to the corresponding old predicate. For instance, the translation of (4) is

$$
\operatorname{CIRC}[C(\Pi)] \wedge \forall X\left(q^{\prime}(X) \leftrightarrow q(X)\right) ;
$$

the circumscription operator CIRC is understood here as the minimization of the extents of $p, q, r$.

The stable models of $\Pi$ can be characterized as the Herbrand interpretations satisfying the translation of $\Pi$, with the new (primed) predicates removed from them ("forgotten").

An interesting feature of this Definition $F$ is that, unlike Definitions A-E, it does not involve grounding. We can ask what non-Herbrand models of the translation of a logic program look like. Can it be convenient in some cases to 
represent possible states of affairs by such "non-Herbrand stable models" of a logic program? A non-Herbrand model may include an object that is different from the values of all ground terms, or there may be several ground terms having the same value in it; can this be sometimes useful?

We will return to the relationship between stable models and circumscription in Section 11.

\section{Definitions $G$ and $H$, in Terms of Tightening and the Situation Calculus}

We will talk now about two characterizations of stable models that are based, like Definition F, on translations into classical logic that use auxiliary predicates.

For a class of logic programs called tight, stable models are identical to Herbrand models of the program's completion [Fages, 1991]. (Programs (2) and (3), used above to illustrate peculiarities of the completion semantics, are not tight.) Definition $G$ [Wallace, 1993] is based on a process of "tightening" that makes an arbitrary traditional program tight. This process uses two auxiliary symbols: the object constant 0 and the unary function constant $s$ ("successor"). Besides, the tightened program uses auxiliary predicates with an additional numeric argument. Intuitively, $p(X, N)$ expresses that there exists a sequence of $N$ "applications" of rules of the program that "establishes" $p(X)$. The stable models of a program are described then as Herbrand models of the completion of the result of its tightening, with the auxiliary symbols "forgotten."

We will not reproduce here the definition of tightening, but here is an example: the result of tightening program (4) is

$$
\begin{aligned}
& p(a, s(N)) . \quad p(b, s(N)) . \quad q(a, s(N)) . \\
& r(X, s(N)) \leftarrow p(X, N), \text { not } q(X) . \\
& p(X) \leftarrow p(X, N) . \\
& q(X) \leftarrow q(X, N) . \\
& r(X) \leftarrow r(X, N) .
\end{aligned}
$$

Rules in line 1 tell us that $p(a)$ can be established in any number of steps that is greater than 0 ; similarly for $p(b)$ and $q(a)$. According to line $2, r(X)$ can be established in $N+1$ steps if $p(X)$ can be established in $N$ steps and $q(X)$ cannot be established at all (note that an occurrence of a predicate does not get an additional numeric argument if it is negated). Finally, an atom holds if it can be established by some number $N$ of rule applications.

Definition $H$ [Lin and Reiter, 1997] treats a rule in a logic program as an abbreviated description of the effect of an action - the action of "applying" that rule - in the situation calculus. ${ }^{3}$ For instance, if the action corresponding to the last rule of $(4)$ is denoted by lastrule $(X)$ then that rule can be viewed as

${ }^{3}$ See [Reiter, 2001] for a detailed description of the situation calculus [McCarthy and Hayes, 1969] as developed by the Toronto school. 
shorthand for the situation calculus formula

$$
p(X, S) \wedge \neg \exists S(q(X, S)) \rightarrow r(X, \operatorname{do}(\text { lastrule }(X), S))
$$

(if $p(X)$ holds in situation $S$ and $q(X)$ does not hold in any situation then $r(X)$ holds after executing action lastrule $(X)$ in situation $S$ ).

In this approach to stable models, the situation calculus function do plays the same role as adding 1 to $N$ in Wallace's theory. Instead of program completion, Lin and Reiter use the process of turning effect axioms into successor state axioms, which is standard in applications of the situation calculus.

\section{Definition I, in Terms of Equilibrium Logic}

The logic of here-and-there, going back to the early days of modern logic [Heyting, 1930], is a modification of classical propositional logic in which propositional interpretations in the usual sense - assignments, or sets of atoms - are replaced by pairs $(X, Y)$ of sets of atoms such that $X \subseteq Y$. (We think of $X$ as the set of atoms that are true "here", and $Y$ as the set of the atoms that are true "there.") The semantics of this logic defines when $(X, Y)$ satisfies a formula $F$.

In [Pearce, 1997], the logic of here-and-there was used as a starting point for defining a nonmonotonic logic closely related to stable models. According to Pearce, a pair $(Y, Y)$ is an equilibrium model of a propositional formula $F$ if $F$ is satisfied in the logic of here-and-there by $(Y, Y)$ but is not satisfied by $(X, Y)$ for any proper subset $X$ of $Y$. Pearce showed that a set $M$ of atoms is a stable model of a program $\Pi$ iff $(M, M)$ is an equilibrium model of the set of propositional formulas (8) corresponding to the grounded rules of $\Pi$.

This Definition $I$ is important for two reasons. First, it suggests a way to extend the concept of a stable model from traditional rules - formulas of form (1) to arbitrary propositional formulas: we can say that $M$ is a stable model of a propositional formula $F$ if $(M, M)$ is an equilibrium model of $F$. This is valuable from the perspective of answer set programming, because many "nonstandard" constructs commonly used in ASP programs, such as choice rules and weight constraints, can be viewed as abbreviations for propositional formulas [Ferraris and Lifschitz, 2005]. Second, Definition I is a key to the theorem about the relationship between the concept of strong equivalence and the logic of here-and-there [Lifschitz et al., 2001].

\section{Definitions $\mathbf{J}$ and $\mathrm{K}$, in Terms of Modified Reducts}

Faber et al. [2004] modified the definition of the reduct reproduced in Section 5 by including the positive members of the body, along with negative members, in the description of step (i), and by removing step (ii) altogeher. In other words, in the modified process of constructing the reduct relative to $M$ we delete from the program all rules (1) containing in their bodies a term $A_{i}$ such that $A_{i} \notin M$ 
or a term not $A_{i}$ such that $A_{i} \in M$; the other rules of the program remain unchanged. For instance, the modified reduct of program (4) relative to (5) is

$$
\begin{aligned}
& p(a) . \quad p(b) . \quad q(a) . \\
& r(b) \leftarrow p(b), \text { not } q(b) .
\end{aligned}
$$

Unlike the reduct (11), this modified reduct contains negation as failure in the last rule. Generally, unlike the reduct in the sense of Section 5, the modified reduct of a program has several minimal models.

According to Definition $J, M$ is a stable model of $\Pi$ iff $M$ is a minimal model of the modified reduct of $\Pi$ relative to $M$.

Ferraris [2005] modified the definition of the reduct in a different way. The reduct of a program $\Pi$ in the sense of Ferraris is obtained from the formulas (8) corresponding to the grounding rules of $\Pi$ by replacing every maximal subformula of $F$ that is not satisfied by $M$ with "false". For instance, the formulas corresponding to the grounded rules (9) of (4) are the formulas

$$
\begin{aligned}
& p(a), \quad p(b), \quad q(a), \\
& \text { false } \rightarrow \text { false, } \\
& p(b) \wedge \neg \text { false } \rightarrow r(b) .
\end{aligned}
$$

Definition $K$ : $M$ is a stable model of $\Pi$ iff $M$ is a minimal model of the reduct of $\Pi$ in the sense of Ferraris relative to $M$.

Definitions $\mathrm{J}$ and $\mathrm{K}$ are valuable because, like definition I, they can be extended to some nontraditional programs. The former was introduced, in fact, in connection with the problem of extending the stable model semantics to programs with aggregates. The latter provides a satisfactory solution to the problem of aggregates as well. Furthermore, it can be applied in a straightforward way to arbitrary propositional formulas, and this generalization of the stable model semantics turned out to be equivalent to the generalization based on equilibrium logic that was mentioned at the end of Section 9 .

\section{Definition L, in Terms of Modified Circumscription}

Ferraris et al. [2007] defined a modification of circumscription called the stable model operator, SM. According to their Definition L, an Herbrand interpretation $M$ is a stable model of $\Pi$ iff $M$ satisfies $\operatorname{SM}[F]$ for the conjunction $F$ of the universal closures of the formulas (8) corresponding to the rules of $\Pi$.

Syntactically, the difference between SM and circumscription is really minor. If $F$ contains neither implications nor negations then $\mathrm{SM}[F]$ does not differ from CIRC $[F]$ at all. If $F$ has "one level of implications" and no negations (as, for instance, when $F$ corresponds to a set of traditional rules without negation, such as (2) and (3)), $\mathrm{SM}[F]$ is equivalent to $\operatorname{CIRC}[F]$. But SM becomes essentially different from CIRC as soon as we allow negation in the bodies of rules.

The difference between $\mathrm{SM}[F]$ and the formulas used in Definition $\mathrm{F}$ is that the former does not involve auxiliary predicates and consequently does not require additional conjunctive terms relating auxiliary predicates to the predicates occurring in the program. 
Definition L combines the main attractive feature of Definitions F, G, and $\mathrm{H}$ - no need for grounding - with the main attractive feature of Definitions I and $\mathrm{K}$-applicability to formulas of arbitrarily complex logical form. This fact allowed Lee et al. [2008] to give a semantics for an ASP language with choice rules and aggregates without any references to grounding.

Among the other definitions of a stable model discussed in this paper, Definition I, based on equilibrium logic, is the closest relative of Definition L. Indeed, in [Pearce et al., 2001] the semantics of equilibrium logic is expressed by quantified Boolean formulas, and we can say that Definition L eliminated the need to ground the program using the fact that the approach of that paper can be easily extended from propositional formulas to first-order formulas.

A characterization of stable models that involves grounding but is otherwise similar to Definition L is given in [Lin and Zhou, 2007]. It has emerged from research on the nonmonotonic logic of knowledge and justified assumptions [Lin and Zhao, 2002].

\section{Conclusion}

Research on stable models has brought us many pleasant surprises.

At the time when the theory of iterated fixpoints of stratified programs was the best available approach to semantics of logic programming, it was difficult to expect that an alternative as general and as simple as Definition $\mathrm{C}$ would be found. And prior to the invention of Definition K, who could think that Definition $\mathrm{C}$ can be extended to choice rules, aggregates and more without paying any price in terms of the simplicity of the process of constructing the reduct?

A close relationship between stable models and a nonclassical logic that had been invented decades before the emergence of logic programming was a big surprise. The possibility of defining stable models by twisting the definition of circumscription just a little was a surprise too.

There was a time when the completion semantics, the well-founded semantics, and the stable model semantics - and a few others - were seen as rivals; every person interested in the semantics of negation in logic programming would tell you then which one was his favorite. Surprisingly, these bitter rivals turned out to be so closely related to each other on a technical level that they eventually became good friends. One cannot study the algorithms used today for generating stable models without learning first about completion and unfounded sets.

And maybe the biggest surprise of all was that an attempt to clarify some semantic issues related to negation in Prolog was destined to be enriched by computational ideas coming from research on the design of SAT solvers and to give rise to a new knowledge representation paradigm, answer set programming.

\section{Acknowledgements}

Many thanks to Michael Gelfond, Joohyung Lee, Nicola Leone, Yuliya Lierler, Fangzhen Lin,Victor Marek, and Mirek Truszczyński for comments on a draft of 
this note. I am also grateful to Mirek and to Andrea Formisano for the invitation to contribute a paper to the special session on stable models planned as part of ICLP'08. This work was partially supported by the National Science Foundation under Grant IIS-0712113.

\section{References}

[Apt et al., 1988] Krzysztof Apt, Howard Blair, and Adrian Walker. Towards a theory of declarative knowledge. In Jack Minker, editor, Foundations of Deductive Databases and Logic Programming, pages 89-148. Morgan Kaufmann, San Mateo, CA, 1988.

[Balduccini and Gelfond, 2003] Marcello Balduccini and Michael Gelfond. Logic programs with consistency-restoring rules. ${ }^{4}$ In Working Notes of the AAAI Spring Symposium on Logical Formalizations of Commonsense Reasoning, 2003.

[Bidoit and Froidevaux, 1987] Nicole Bidoit and Christine Froidevaux. Minimalism subsumes default logic and circumscription in stratified logic programming. In Proc. LICS-87, pages 89-97, 1987.

[Clark, 1978] Keith Clark. Negation as failure. In Herve Gallaire and Jack Minker, editors, Logic and Data Bases, pages 293-322. Plenum Press, New York, 1978.

[Faber et al., 2004] Wolfgang Faber, Nicola Leone, and Gerard Pfeifer. Recursive aggregates in disjunctive logic programs: Semantics and complexity. ${ }^{5}$ In Proceedings of European Conference on Logics in Artificial Intelligence (JELIA), 2004.

[Fages, 1991] François Fages. A fixpoint semantics for general logic programs compared with the well-supported and stable model semantics. New Generation Computing, 9:425-443, 1991.

[Ferraris and Lifschitz, 2005] Paolo Ferraris and Vladimir Lifschitz. Weight constraints as nested expressions. Theory and Practice of Logic Programming, 5:45-74, 2005.

[Ferraris et al., 2007] Paolo Ferraris, Joohyung Lee, and Vladimir Lifschitz. A new perspective on stable models. In Proceedings of International Joint Conference on Artificial Intelligence (IJCAI), pages 372-379, 2007.

[Ferraris, 2005] Paolo Ferraris. Answer sets for propositional theories. In Proceedings of International Conference on Logic Programming and Nonmonotonic Reasoning (LPNMR), pages 119-131, 2005.

[Fine, 1989] Kit Fine. The justification of negation as failure. In Proceedings of the Eighth International Congress of Logic, Methodology and Philosophy of Science, pages 263-301. North Holland, 1989.

[Gebser et al., 2007] Martin Gebser, Lengning Liu, Gayathri Namasivayam, André Neumann, Torsten Schaub, and Mirosław Truszczynski. The first answer set programming system competition. In Proceedings of the International Conference on Logic Programming and Nonmonotonic Reasoning (LPNMR), pages 3-17. SpringerVerlag, 2007.

[Gelfond and Lifschitz, 1988] Michael Gelfond and Vladimir Lifschitz. The stable model semantics for logic programming. In Robert Kowalski and Kenneth Bowen, editors, Proceedings of International Logic Programming Conference and Symposium, pages 1070-1080. MIT Press, 1988.

[Gelfond and Lifschitz, 1990] Michael Gelfond and Vladimir Lifschitz. Logic programs with classical negation. In David Warren and Peter Szeredi, editors, Proceedings of International Conference on Logic Programming (ICLP), pages 579-597, 1990.

\footnotetext{
${ }^{4}$ http://www.krlab.cs.ttu.edu/papers/download/bg03.pdf .

${ }^{5}$ Revised version: http://www.wfaber.com/research/papers/jelia2004.pdf .
} 
[Gelfond, 1987] Michael Gelfond. On stratified autoepistemic theories. In Proceedings of National Conference on Artificial Intelligence (AAAI), pages 207-211, 1987.

[Heyting, 1930] Arend Heyting. Die formalen Regeln der intuitionistischen Logik. Sitzunsberichte der Preussischen Akademie von Wissenschaften. Physikalischmathematische Klasse, pages 42-56, 1930.

[Lee et al., 2008] Joohyung Lee, Vladimir Lifschitz, and Ravi Palla. A reductive semantics for counting and choice in answer set programming. In Proceedings of the AAAI Conference on Artificial Intelligence (AAAI), pages 472-479, 2008.

[Lee, 2005] Joohyung Lee. A model-theoretic counterpart of loop formulas. In Proceedings of International Joint Conference on Artificial Intelligence (IJCAI), pages 503-508, 2005.

[Lifschitz et al., 1999] Vladimir Lifschitz, Lappoon R. Tang, and Hudson Turner. Nested expressions in logic programs. Annals of Mathematics and Artificial Intelligence, 25:369-389, 1999.

[Lifschitz et al., 2001] Vladimir Lifschitz, David Pearce, and Agustin Valverde. Strongly equivalent logic programs. ACM Transactions on Computational Logic, 2:526-541, 2001.

[Lifschitz, 1988] Vladimir Lifschitz. On the declarative semantics of logic programs with negation. In Jack Minker, editor, Foundations of Deductive Databases and Logic Programming, pages 177-192. Morgan Kaufmann, San Mateo, CA, 1988.

[Lin and Reiter, 1997] Fangzhen Lin and Raymond Reiter. Rules as actions: A situation calculus semantics for logic programs. Journal of Logic Programming, 31:299330, 1997.

[Lin and Zhao, 2002] Fangzhen Lin and Yuting Zhao. ASSAT: Computing answer sets of a logic program by SAT solvers. In Proceedings of National Conference on Artificial Intelligence (AAAI), pages 112-117, 2002.

[Lin and Zhao, 2004] Fangzhen Lin and Yuting Zhao. ASSAT: Computing answer sets of a logic program by SAT solvers. ${ }^{6}$ Artificial Intelligence, 157:115-137, 2004.

[Lin and Zhou, 2007] Fangzhen Lin and Yi Zhou. From answer set logic programming to circumscription via logic of GK. In Proceedings of International Joint Conference on Artificial Intelligence (IJCAI), 2007.

[Lin, 1991] Fangzhen Lin. A Study of Nonmonotonic Reasoning. PhD thesis, Stanford University, 1991.

[Marek and Truszczyński, 1989] Victor Marek and Mirosław Truszczyński. Stable semantics for logic programs and default theories. In Proc. North American Conf. on Logic Programming, pages 243-256, 1989.

[McCarthy and Hayes, 1969] John McCarthy and Patrick Hayes. Some philosophical problems from the standpoint of artificial intelligence. In B. Meltzer and D. Michie, editors, Machine Intelligence, volume 4, pages 463-502. Edinburgh University Press, Edinburgh, 1969.

[McCarthy, 1980] John McCarthy. Circumscription-a form of non-monotonic reasoning. Artificial Intelligence, 13:27-39,171-172, 1980.

[McCarthy, 1986] John McCarthy. Applications of circumscription to formalizing common sense knowledge. Artificial Intelligence, 26(3):89-116, 1986.

[McDermott and Doyle, 1980] Drew McDermott and Jon Doyle. Nonmonotonic logic I. Artificial Intelligence, 13:41-72, 1980.

[McDermott, 1982] Drew McDermott. Nonmonotonic logic II: Nonmonotonic modal theories. Journal of ACM, 29(1):33-57, 1982.

${ }^{6}$ Revised version: http://www.cs.ust.hk/faculty/flin/papers/assat-aij-revised .pdf . 
[Moore, 1985] Robert Moore. Semantical considerations on nonmonotonic logic. Artificial Intelligence, 25(1):75-94, 1985.

[Niemelä and Simons, 2000] Ilkka Niemelä and Patrik Simons. Extending the Smodels system with cardinality and weight constraints. In Jack Minker, editor, Logic-Based Artificial Intelligence, pages 491-521. Kluwer, 2000.

[Pearce et al., 2001] David Pearce, Hans Tompits, and Stefan Woltran. Encodings for equilibrium logic and logic programs with nested expressions. In Proceedings of Portuguese Conference on Artificial Intelligence (EPIA), pages 306-320, 2001.

[Pearce, 1997] David Pearce. A new logical characterization of stable models and answer sets. In Jürgen Dix, Luis Pereira, and Teodor Przymusinski, editors, NonMonotonic Extensions of Logic Programming (Lecture Notes in Artificial Intelligence 1216), pages 57-70. Springer-Verlag, 1997.

[Reiter, 1980] Raymond Reiter. A logic for default reasoning. Artificial Intelligence, 13:81-132, 1980.

[Reiter, 1982] Raymond Reiter. Circumscription implies predicate completion (sometimes). In Proceedings of International Joint Conference on Artificial Intelligence (IJCAI), pages 418-420, 1982.

[Reiter, 2001] Raymond Reiter. Knowledge in Action: Logical Foundations for Specifying and Implementing Dynamical Systems. MIT Press, 2001.

[Saccá and Zaniolo, 1990] Domenico Saccá and Carlo Zaniolo. Stable models and nondeterminism in logic programs with negation. In Proceedings of ACM Symposium on Principles of Database Systems (PODS), pages 205-217, 1990.

[van Emden and Clark, 1984] Maarten van Emden and Keith Clark. The logic of twoperson games. In Micro-PROLOG: Programming in Logic, pages 320-340. PrenticeHall, 1984.

[van Emden and Kowalski, 1976] Maarten van Emden and Robert Kowalski. The semantics of predicate logic as a programming language. Journal of ACM, 23(4):733$742,1976$.

[Van Gelder et al., 1988] Allen Van Gelder, Kenneth A. Ross, and John S. Schlipf. Unfounded sets and well-founded semantics for general logic programs. In Proceedings of the Seventh ACM SIGACT-SIGMOD-SIGART Symposium on Principles of Database Systems, March 21-23, 1988, Austin, Texas, pages 221-230. ACM Press, 1988.

[Van Gelder et al., 1991] Allen Van Gelder, Kenneth Ross, and John Schlipf. The wellfounded semantics for general logic programs. Journal of ACM, 38(3):620-650, 1991.

[Van Gelder, 1988] Allen Van Gelder. Negation as failure using tight derivations for general logic programs. In Jack Minker, editor, Foundations of Deductive Databases and Logic Programming, pages 149-176. Morgan Kaufmann, San Mateo, CA, 1988.

[Wallace, 1993] Mark Wallace. Tight, consistent and computable completions for unrestricted logic programs. Journal of Logic Programming, 15:243-273, 1993. 